\title{
A Comparative Study of Root System Architecture in Seedlings of Brachypodium spp. Using Three Plant Growth Supports
}

\author{
J.M. GonZÁlez*, E. Friero, L. Selfa, S. Froilán and N. Jouve \\ Department of Biomedicine y Biotechnology, Edifice of Cell Biology and Genetics. University of Alcalá, \\ 28871 Alcalá de Henares (Madrid), Spain
}

(Received 14 April 2015; Accepted 3 June 2015;

Communicated by J. Zhang)

\begin{abstract}
This work reports a rapid procedure of comparing root development among different genotypes of Brachypodium spp., using three plant growth supports: gel chamber, 'Termita' chamber and Whatman paper. Eight variables of the root system architecture (RSA) (number of seminal roots, number of lateral roots, total length of the roots, length of the primary root, mean diameter of the roots, mean diameter of the primary root, total surface area and total volume of the roots) were studied in seedling of four genotypes each of Brachypodium distachyon, B. stacei and B. hybridum. Correlations between pairs of growth supports in terms of the eight variables examined were highly significant. In all three supports, B. stacei showed the greatest root system development while $B$. distachyon showed the least; $B$. hybri$d u m$, an allotetraploid species derived from hybridization between $B$. distachyon and B. stacei, showed intermediate development. ANOVA and LSD tests showed that significant differences exist between the supports, species and genotypes with respect to all the variables analysed. A cluster analysis was conducted to determine if the RSA traits could be used to differentiate the species and genotypes of Brachypodium. This analysis allowed differentiated between the three species and twelve genotypes of Brachypodium spp., although a certain overlap between species was observed. The Whatman paper support was the easiest to use, and is recommended for the characterization of large collections of genotypes.
\end{abstract}

Keywords: Brachypodium distachyon; stacei, hybridum, RSA, variability

\section{Introduction}

It has been proposed that the genetic improvement of the root system architecture of plants (RSA), (Lynch 1995), might increase crop yields and plant tolerance to abiotic stresses. For example, encouraging the growth of deeper roots may increase tolerance to drought (Reynolds et al. 2007; Wasson et al. 2012), whereas encouraging more superficial roots might increase the capacity to grow in soils poor in phosphate (Gahoonia and Nielsen 2004; Zhu et al. 2005; White et al. 2013).

The RSA is very dynamic, varies between species and genotypes, and is affected by the composition and type of soil, the amount of water available, etc. (Forde and Lorenzo 2001; White et al. 2013). Root development was analysed in plants grown in the field

*Corresponding author; E-mail: juanm.gonzalez@uah.es 
(Trachsel et al. 2011), in pots filled with sand or soil (Waines and Ehdaie 2007) and in rhizotrons (Tyagi et al. 2014; Ytting et al. 2014). In all these studies, however, the roots had to be extracted and washed before any phenotypic examination could be made. More sophisticated methods involving X-ray microcomputed tomography images allow analyses to be made without removing the roots from their substrate, but only a few laboratories have the necessary equipment, and only very small numbers of plants can be studied (Tracy et al. 2010; Mairhofer et al. 2012).

Reliable methods that allow the easy examination of growing roots must, therefore, be developed if RSA improvement programs are to be undertaken. To date, gelled nutrient media (Benboroug 2004; Sanguinetti et al. 2007; Manschadi et al. 2008), blotting paper (Zhu et al. 2005; Richard et al. 2015) and hydroponic culture systems (Ren et al. 2012; Obara et al. 2014) have all been used to provide artificial support for such purposes in the laboratory. In some cases these methods have helped identify QTL related to characteristics of the root system of species such as maize, wheat and rice, etc. (Price et. al. 1997; Ren et al. 2012; Christopher et al. 2013; Canè et al. 2014). Marker-assisted selection (MAS) has also been used to introduce QTL that control different RSA characteristics in some rice varieties into the genomes of other rice varieties (Steele et al. 2006, 2013).

$B$. distachyon is a wild species that serves as model for cereals cultivated in moderate climates (Draper et al. 2001) and its genome has been sequenced (IBI 2010). Until recently, it was considered that $B$. distachyon was integrated by three cytotypes with $2 \mathrm{n}=10,20$ and 30. The taxonomic classification has been reviewed by Catalán et al. (2012) and has been proposed to each cytotypes a different species named as B. distachyon $(2 \mathrm{n}=10)$, B. stacei $(2 \mathrm{n}=20)$ and B. hybridum that is an allotetraploid $(2 \mathrm{n}=30$, $x=5+x=10)$ derived from the cross between the above two. The results of basic and applied research into these three species and specially in $B$. distachyon, will serve as a reference to allow genetic improvements to be made in cereals of greater economic importance, such as wheat, barley, oats, etc. (Watt et al. 2009; Chochois et al. 2012).

In the present work, three plant growth supports (PGS): gel chamber, 'Termita' chamber and Whatman paper, were used to study the phenotypes of eight RSA characteristics in four genotypes each of B. distachyon, B. stacei and B. hybridum. The aims of the work were: a) to test the above three plant growth supports as means of allowing the inspection of RSA during early root development; b) to identify differences between and within the RSA of the different species and genotypes of Brachypodium, c) to select the plant growth support system that most easily allows differences between genotypes to be identified, with a view to its use in RSA genetic improvement programs, and d) to determine whether the characteristics studied are of use in classifying the genotypes and species analysed.

\section{Materials and Methods}

\section{Plant materials}

The plant materials examined included four genotypes each of $B$. distachyon $(2 \mathrm{n}=10)$ (including genotype Bd-21, the full genome of which has been sequenced by the International Brachypodium Initiative [IBI 2010]), B. stacei $(2 \mathrm{n}=20)$ and B. hybridum $(2 \mathrm{n}=30)$. 
These genotypes all originated from single plants belonging to 11 wild populations (three of $B$. dystachyon and four each of $B$. stacei and B. hybridum), harvested in different places around the Iberian Peninsula (Hammami et al. 2011) (Table S1*).

Sixty grains of similar size were selected for each of the genotypes, and surface sterilized for $20 \mathrm{~min}$ in $10 \%$ calcium hypochlorite. They were then rinsed three times with sterile deionised water, spread over six Petri dishes containing blotting paper and $7 \mathrm{ml}$ of deionised sterile water, and kept at $24{ }^{\circ} \mathrm{C}$ for 1 day, at $4{ }^{\circ} \mathrm{C}$ for the next 2 days, and at $24{ }^{\circ} \mathrm{C}$ for the next 2 days. Four replicates of five seeds of each genotype were then placed on three different PGS: a) gel chamber, b) 'Termita' chamber, and c) Whatman paper.

\section{Gel chamber}

These were constructed using two glass plates, each measuring $210 \times 290 \times 2.5 \mathrm{~mm}$, separated from each other by a U-shaped piece of methacrylate (width $10 \mathrm{~mm}$, height $3 \mathrm{~mm}$ ) (Fig. S1a). The chamber was filled with Aniol (1984) mineral solution, plus $0.75 \mathrm{~g} / \mathrm{l}$ of Phyto Agar (Duchefa, P 1003), and $500 \mu \mathrm{l} / 1$ Plant Preservative Mixture ${ }^{\mathrm{TM}}$ (PPM) (Plant Cell Technology, Inc., Washington DC) to prevent microbial or fungi contamination. After adjustment to $\mathrm{pH} 5.8$, the solution was sterilized at $121{ }^{\circ} \mathrm{C}$ for $20 \mathrm{~min}$ before being poured into the chamber. On the top of the gel, five uniformly spaced $10 \mathrm{~mm}$-deep holes were made, and one seed planted per hole. The chambers were then covered with two sheets of black plastic to protect the roots from the light.

\section{'Termita' chamber}

These chambers were constructed in a similar manner to the gel chamber, but using a U-shaped piece of polystyrene (width $10 \mathrm{~mm}$, height $30 \mathrm{~mm}$ ), with three $5 \mathrm{~mm}$ holes in its lower part to drain off excess nutrient solution. The chambers were filled with 'Termita' (exfoliated vermiculite) (Asfaltex, S.A., Barcelona, http://www.asfaltex.com/) and soaked with $250 \mathrm{ml}$ Aniol solution and $500 \mu \mathrm{l} / 1 \mathrm{PPM}^{\mathrm{TM}}$ (Fig. S1b). The $\mathrm{pH}$ was adjusted to 5.8. At the top, five $10 \mathrm{~mm}$-deep holes were made and one seed sown per hole. The chambers were then covered with two sheets of black plastic.

\section{Whatman paper}

A sheet of sterilized Whatman paper ( $3 \mathrm{~mm}$ CHR chromatography paper) $(210 \times 290$ $\mathrm{mm}$ ) was placed on the surface of a glass plate of the same dimensions. Five $10 \mathrm{~mm}$-deep and $5 \mathrm{~mm}$-wide nicks were made at the top of the paper to make places to position the grains (Fig. S1c). The seeds and Whatman paper sheet were then covered with a piece of filter paper and two black plastic sheets (front and on the back) to keep the roots in the dark. The entire system was secured using clips at the corners, placed vertically in a plastic container and submerged $30 \mathrm{~mm}$ in Aniol solution and $500 \mu \mathrm{l} / 1$ of $\mathrm{PPM}^{\mathrm{TM}}(\mathrm{pH}=5.8)$.

*Further details about the Electronic Supplementary Material (ESM) can be found at the end of the article. 
All three PGS were placed in a chamber at a temperature of $18-22{ }^{\circ} \mathrm{C}$ under a $12 \mathrm{~h}$ light/dark photoperiod. After 15 days the plantlets showed one or two leaves and the roots have developed in all three supports (Fig. S1, d, e, f). The roots developed from every seed were removed from the support systems, rinsed, and spread out on a glass plate. They were then scanned at 300 ppi using a Hewlett-Packard Scanjet 5300C scanner. The images produced were saved in jpg format at maximum quality. A total of 14736 seminal and lateral roots were studied.

\section{Root analysis}

The root images were analysed using SmartRoot software v.3.32 (Lobet et al. 2011), and ImageJ1.46R software (http://imagej.nih.gov/ij/download.html). Root types were identified and measured using the manual and semi-automated SmartRoot procedures. The number of seminal and lateral roots of each grains, and the length, mean diameter, surface area and volume of each root were then recorded in a Microsoft Access file. The data for these variables were then exported to a Microsoft Excel file and the following variables were recorded: the number of seminal roots including the primary root (nPR), the number of lateral roots $(\mathrm{nLR})$, the total length of the roots $(\mathrm{L})$ in $\mathrm{cm}$, the length of the primary root (LR) in $\mathrm{cm}$, the mean diameter of the roots (D) in $\mathrm{cm}$, the mean diameter of the primary root (DPR) in cm, the total surface area (S) in $\mathrm{cm}^{2}$, and the total volume (V) of the roots in $\mathrm{cm}^{3}$. Means for these variables were recorded by genotype, PGS, and replica.

\section{Statistical analysis}

Correlations using the Spearman correlation coefficient, have been calculated between pairs of PGS, considering the mean values of the eight variables. ANOVA was used to confirm the existence of differences between the species, genotypes and PGS in terms of the values of the variables analysed. Before ANOVA analysis, all data were transformed by $\sqrt{ } \mathrm{x}$ in order to normalise the distribution. The least squares difference (LSD) test was used for comparing means. Euclidean distance using the mean of each variable, obtained as a mean value of the three PGS was calculated and cluster analysis based on the nearest neighbour algorithm was performed to construct a dendrogram. All analyses were performed using STATGRAPHICS Plus v.5.1 software.

\section{Results}

\section{Overall comparison of the three PGS}

The mean values of the analysed variables were determined for all Brachypodium species together in each of the PGS. In general, the variables reach the lowest and highest values in the gel chamber and Whatman paper respectively, except in the case of the variable nPR (Fig. 1a). Considering the mean values of each of the eight variables, the Spearman 
correlation coefficients were calculated between pairs of growth support, being always higher than $r=0.96$ and highly significant $(p<0.001)$. In order to determine if the variables have different behaviour in the three PGS, ANOVAs were carried out, considering the four replicates and taking as independent factor the type of PGS. In all cases, significant differences were detected $(p<0.05)$ and Table S2a shows the results of LSD tests, identifying exactly where these differences lay.

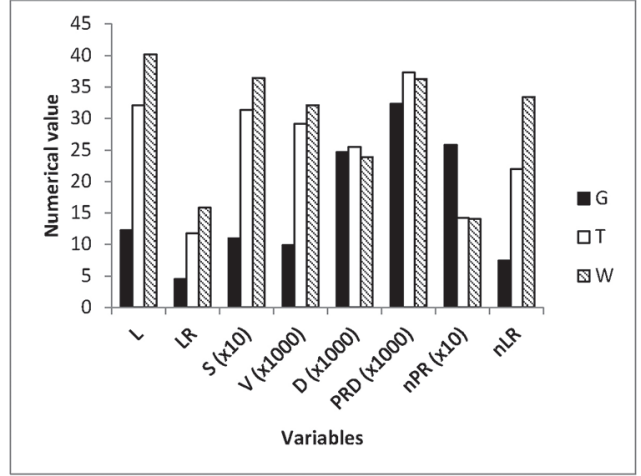

a)

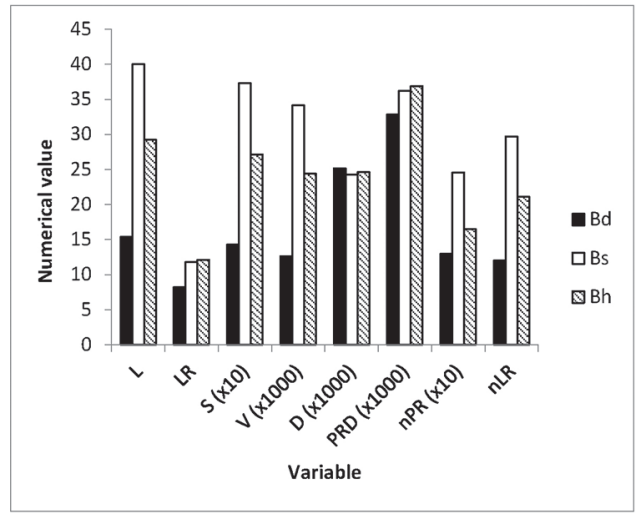

b)

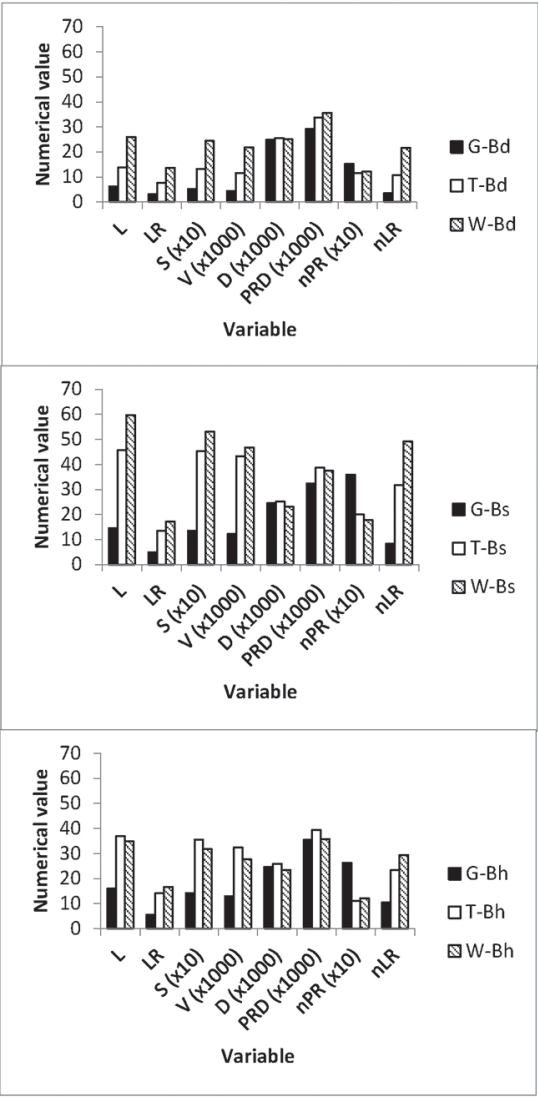

c)

Figure 1. Mean values of the eight variables recorded in: a) each type of PGS for all species of Brachypodium taken together; b) each individual species of Brachypodium taken together the results of the three PGS; c) each of the three types of PGS and for each species or Brachypodium. L, total length of the roots in cm; LR, length of the primary root in $\mathrm{cm} ; \mathrm{S}$, total surface area of the roots in $\mathrm{cm}^{2} ; \mathrm{V}$, total volume of the roots in $\mathrm{cm}^{3} ; \mathrm{D}$, mean diameter of the roots in $\mathrm{cm}$; PRD, mean diameter of the primary roots in $\mathrm{cm}$; $\mathrm{nPR}$, number of seminal roots; nLR, number of lateral roots. G: growth in gel chamber; T: growth in 'Termita' chamber; W: growth on Whatman paper. Bd: B. distachyon; Bs: B. stacei and Bh: B. hybridum 


\section{Comparison of root system development in the Brachypodium species}

Considering simultaneously the three PGS for each species of Brachypodium, the mean values of each variable were calculated. B. stacei and B. hybridum are the species that have shown the highest development (Fig. 1b). Taking the species as the independent factor, ANOVA for each variable were calculated and significant differences $(p<0.05)$ were detected in all cases. The results of the LSD tests are shown in Table S2b.

\section{Comparison of the three PGS with respect to each of the species of Brachypodium}

For each separate species of Brachypodium, the means of the eight variables recorded for each of the PGS were calculated and shown in Fig. 1c. B. distachyon showed the smallest root development in all PGS, followed by B. hybridum and B. stacei. For each species and variable and taking the type of PGS as independent factor, ANOVAs were calculated showing significant differences $(p<0.05)$ in all cases. Table S2c shows the results of the LSD test, which identify these differences.

\section{Intergenotypic variability of the root system characteristics}

In each of the species, were calculated ANOVAs for each variable, taking to the genotype as independent factor. ANOVAs showed the four genotypes of each Brachypodium species to differ significantly $(p<0.05)$ with respect to every variable analysed. The LSD tests identified where these differences lay (Table S2d).

\section{Cluster analysis}

To determine whether the characteristics of the RSA can be used to differentiate the species and genotypes of Brachypodium, the similarity between genotypes was assessed considering the mean of each variable, obtained as a mean value of the three PGS. Euclidean distance using these values was calculated and cluster analysis based on the nearest neighbour algorithm was performed to construct a dendrogram (Fig. S2).

\section{Discussion}

To understand the genetic variation of any complex characteristic related to agricultural production, rapid and precise procedures for analyzing plant phenotypes are required (Cobb et al. 2013). The root system is one of the main factors influencing yield, since the capture of water and nutrients, and the support and anchorage of the stems, all depend on the roots. The degree of development of the roots and the RSA can vary widely depending on the species, the genotype and the availability of water and nutrients (Forde and Lorenzo 2001; Manschadi et al. 2008; Ingram et al. 2012; Christopher et al. 2013; Obara et al. 2014; Tyagi et al. 2014), but its study is difficult since the roots grow underground and are hidden from direct observation. However, laboratory procedures of visualizing the 
RSA exists. One of the main problems of using artificial PGS for characterizing the root phenotype is that root development might not be the same as in the ground. Nevertheless, studies in bean, maize, rice and soybean all indicate a relationship that exists between the results obtained for different RSA characteristics in the field and in artificial systems (Bengough et al. 2004; Sanguineti et al. 2007; Zhu et al. 2011; Canè et al. 2014).

The present work shows that, for all three species of Brachypodium taken as a whole, the type of PGS (Fig. 1a) influences the degree of growth reached by the roots. Similarly, when the species of Brachypodium are analysed separately, the values of the eight variables measured depend on the PGS used (Fig. 2c). Thus, in B. distachyon and B. stacei, most of the variables returned higher values with the Whatman paper support, followed by the 'Termita' chamber and gel chamber supports. Nevertheless, in B. hybridum, the development of the roots in the 'Termita' chamber and Whatman paper systems was very similar, and greater than that observed in the gel chambers. In contrast, the number seminal roots was greatest for all three species in the gel chamber system. This might be due to the anoxic environment provided by this PGS by some unknown mechanism that might induce the formation of a larger number of seminal roots. Hargreaves et al. (2009) obtained similar results in another characters of barley, finding that plants developed roots of larger diameter and longer total length when grown in gel chambers than in natural soil.

The differences observed in the development of the RSA according to the type of PGS used, agree with results obtained in earlier studies in which the root system was noted to be very plastic and adaptable to the environment in which the plant was grown (Forde and Lorenzo 2001; Zhu et al. 2005; White et al. 2013; Tyagi et al. 2014). In the present work, the 'Termita' chamber was probably the PGS that best reflected the development of the roots in natural soil (at least from a visual point of view), being the means of most of the analysed variables recorded for this support system were between those returned by the other two systems (Fig. 1a and c). Nevertheless, the positive correlation between the different RSA variables across the PGS shows that any can be used in analyses of the root system. However, when characterizing the RSA of different species, or genotypes of the same species, the same PGS should be used in all the experiments. Different PGS could be used, however, to highlight the differences in particular characters, because one PGS or another can best reveal them. From our experience in this work, the Whatman paper support is the simplest to set up, and this PGS has the potential to allow the rapid, costeffective characterization of many types of plant material. It thus allows the best genotypes to be selected for use in genetic improvement programs aimed at obtaining new varieties adapted to deficits or excesses of $\mathrm{N}, \mathrm{PO}_{4}, \mathrm{Fe}, \mathrm{Al}, \mathrm{NaCl}$ and water.

The selection and improvement of root system requires information on interspecific and intergenotypic variability. Different authors have reported intergenotypic differences for several characteristics of the root system of rice (Price et al. 1997), bread wheat (Waines and Ehdaie 2007) or durum wheat (Sanguineti et al. 2007, Canè et al. 2014). Pacheco-Villalobos and Hardtke (2012) observed variation in the RSA of different accessions of Brachypodium and Ingram et al. (2012) studied two accessions of B. distachyon that showed different RSA behaviour in different nutrient media: while one maintained 
the growth of auxiliary roots in low $\mathrm{N}$ conditions, the other maintained lateral root growth in low $\mathrm{P}$ conditions.

In the present work, the results of the ANOVAs and LSD tests (Table S2d) indicated the RSA showed intergenotypic variability in terms of the values of the studied variables recorded for the different PGS. However, it should be noted that genotypes $\mathrm{Bd} 21$ and $\mathrm{Bd} 3113$ of $B$. distachyon were very similar, as were Bs129 and Bs485 of B. stacei, and Bh409 and Bh3107 of B. hybridum (Table S2d). The intergenotypic differences recorded for the different characteristics might be due to different genetic systems, which might allow independent improvement strategies to be designed for each of these characteristics.

Certain RSA characteristics have been used to distinguish between groups of genotypes in other species. Manschadi et al. (2008) performed a cluster analysis based on the angle of growth and number of roots in 29 genotypes of wheat, and were able to differentiate between four groups that reflected different genetic backgrounds and capacities to adapt to the environment. In the cluster analysis performed, the three species of Brachypodium were distinguishable, although a certain overlap between the genotypes were observed (Fig. S2). For instance, genotype Bs115 is more related with three genotypes of B. distachyon than the other three genotypes of B. stacei, Moreover, Bd160 and Bh486, appear clearly separated on the dendrogram, probably due to these genotypes have, respectively, the shortest and the largest radicular system of the genotypes of $B$. distachyon and $B$. hybridum analysed. These results are similar to those obtained by other authors using biochemical and molecular markers to differentiate between the members of a large collection of genotypes belonging to the present three species of Brachypodium (including those studied in the present work) (Hammami et al. 2011, 2014). The overlap observed in the grouping of the genotypes of the three species can be explained by the existing evolutionary proximity among them, being $B$. hybridum an allotetraploid of the other two species.

\section{Acknowledgements}

This study was funded by a grant from the Spanish Ministry of Education and Science (AGL2012-34052). The authors thank Adrian Burton for linguistic assistance.

\section{References}

Aniol, A. 1984. Induction of aluminum tolerance in wheat seedlings by low doses of aluminum in the nutrient solution. Plant Physiol. 75:551-555.

Bengough, A.G., Gordon, D.C., Al-Menaie, H., Ellis, R.P., Allan, D., Keith, R., Thomas, W.T.B., Forster, B.P. 2004. Gel observation chamber for rapid screening of root traits in cereal seedlings. Plant Soil 262:63-70.

Canè, M.A., Maccaferri, M., Nazemi, G., Salvi, S., Francia, R., Colalongo, Ch., Tuberosa, T. 2014. Association mapping for root architectural traits in durum wheat as related to agronomic performance. Mol. Breeding 34:1629-1645.

Catalán, P., Muller, J., Hasterok, R., Jenkins, G., Mur, L.A., Langdon, T., Betekhtin, A., Siwinska, D., Pimentel, M., López-Alvarez, D. 2012. Evolution and taxonomic split of the model grass Brachypodium distachyon. Ann. Bot. 109:385-405. 
Chochois, V., Vogel, J.P., Watt, M. 2012. Application of Brachypodium to the genetic improvement of wheat roots. J. Exp. Bot. 63:3467-3474.

Christopher, J., Christopher, M., Jennings, R., Jones, S., Fletcher, S., Borrell, A., Manschadi, A.M., Jordan, D., Mace, E., Hammer, G. 2013. QTL for root angle and number in a population developed from bread wheats (Triticum aestivum) with contrasting adaptation to water-limited environments. Theor. Appl. Genet. 126:1563-1574.

Cobb, J.N., DeClerck, G., Greenberg, A., Clark, R., McCouch, S.R. 2013. Next-generation phenotyping: requirements and strategies for enhancing our understanding of genotype-phenotype relationships and its relevance to crop improvement. Theor. Appl. Genet. 126:867-887.

Draper, J., Mur, L.A., Jenkins, G., Ghosh-Biswas, G.C., Bablak, P., Hasterok, R., Routledge, A.P.M. 2001. Brachypodium distachyon. A new model system for functional genomics in grasses. Plant Physiol. 127:1539-1555.

Forde, B., Lorenzo, H. 2001. The nutritional control of root development. Plant Soil 232:51-68.

Gahoonia, T.S., Nielsen, N.E. 2004. Root traits as tools for creating phosphorus efficient crop varieties. Plant Soil 260:47-57.

Hammami, R., Jouve, N., Cuadrado, A., Soler, C., González, J.M. 2011. Prolamin storage proteins and alloploidy in wild populations of the small grass Brachypodium distachyon (L.) P. Beauv. Plant Syst. Evol. 297:99-112.

Hammami, R., Jouve, N., Soler, C., Frieiro, E., González, J.M. 2014. Genetic diversity of SSR and ISSR markers in wild populations of Brachypodium distachyon and its close relatives B. stacei and B. hybridum (Poaceae). Plant Syst. Evol. 300:2029-2040.

Hargreaves, C.E., Gregory, P.J., Bengough, A.G. 2009. Measuring root traits in barley (Hordeum vulgare ssp. vulgare and ssp. spontaneum) seedlings using gel chambers, soil sacs and X-ray microtomography. Plant Soil 316:285-297.

IBI 2010. Genome sequencing and analysis of the model grass Brachypodium distachyon. Nature 463:763-768.

Ingram, P.A., Zhu, J., Shariff, A., Davis, I.W., Benfey, P.N., Elich, T. 2012. High-throughput imaging and analysis of root system architecture in Brachypodium distachyon under differential nutrient availability. Phil. Trans. R. Soc. B. 367:1559-1569.

Lobet, G., Pagès, L., Draye, X. 2011. A novel image-analysis toolbox enabling quantitative analysis of root system architecture. Plant Physiol. 157:29-39.

Lynch, J. 1995. Root architecture and plant productivity. Plant Physiol. 109:7-13.

Mairhofer, S., Zappala, S., Tracy, S.R., Sturrock, C., Bennett, M., Mooney, S.J., Pridmore, T. 2012. RooTrak: Automated Recovery of three-dimensional plant root architecture in soil from X-ray microcomputed tomography images using visual tracking. Plant Physiol. 158:561-569.

Manschadi, A.M., Hammer, G.L., Christopher, J.T., deVoi, P. 2008. 1 Genotypic variation in seedling root architectural traits and implications for drought adaptation in wheat (Triticum aestivum L.). Plant Soil 303:115-129.

Obara, M., Ishimaru, T., Abiko, T., Fujita, D., Kobayashi, N., Yanagihara, S., Fukuta, Y. 2014. Identification and characterization of quantitative trait loci for root elongation by using introgression lines with genetic background of Indica-type rice variety IR64. Plant Biotechnol. Rep. 8:267-277.

Pacheco-Villalobos, D., Hardtke, C. 2012. Natural genetic variation of root system architecture from Arabidopsis to Brachypodium: towards adaptive value. Phil. Trans. R. Soc. B. 367:1552-1558.

Price, A.H., Tomos, A.D. 1997. Genetic dissection of root growth in rice (Oryza sativa L.). II: mapping quantitative trait loci using molecular markers. Theor. Appl. Genet. 95:143-152.

Ren, Y., He, X., Liu, D., Li, J., Zhao, X., Li, B., Tong, Y., Zhang, A., Li, Z. 2012. Major quantitative trait loci for seminal root morphology of wheat seedlings. Mol. Breeding 30:139-148.

Reynolds, M., Dreccer, F., Trethowan, R. 2007. Drought-adaptive traits derived from heat wild relatives and landraces. J. Exp. Bot. 58:177-186.

Richard, C.A., Hickey L.T., Fletcher, S., Jennings R., Chenu, K., Christopher J.T. 2015. High-throughput phenotyping of seminal root traits in wheat. Plant Methods: 11:13.

Sanguineti, M.C., Li, S., Maccaferri, M., Corneti, S., Rotondo, F., Chiari, T., Tuberosa, R. 2007. Genetic dissection of seminal root architecture in elite durum wheat germplasm. Ann. Appl. Biol. 151:291-305. 
Steele, K.A., Price, A.H., Shashidhar, H.E., Witcombe, J.R. 2006. Marker-assisted selection to introgress rice QTLs controlling root traits into an Indian upland rice variety. Theor. Appl. Genet. 112:208-221.

Steele, K.A., Price, A.H., Witcombe, J.R., Shrestha, R., Singh, B.N., Gibbons, J.M., Virk, D.S. 2013. QTLs associated with root traits increase yield in upland rice when transferred through marker-assisted selection. Theor. Appl. Genet. 126:101-108.

Trachsel, S., Kaeppler, S.M., Brown, K.M., Lynch, J.P. 2011. Shovelomics: high throughput genotyping of maize (Zea mays L.) root architecture in the field. Plant Soil 341:75-87.

Tracy, S.R., Roberts, J.A., Black, C.R., McNeill, A., Davidson, R., Mooney, S.J. 2010. The X-factor: visualizing undisturbed root architecture in soils using X-ray computed tomography. J. Exp. Bot. 61:311-313.

Tyagi, K., Lee, H.J., Lee, C.A., Steffenson, B.J., Kim, Y.J., Yun, S.J. 2014. Variation in seedling root traits in wild barley (Hordeum vulgare L. ssp. spontaneum) germplasm. Plant Genet. Resources: Characterization and Utilization 12(Suppl.1):S79-S82.

Waines, G., Ehdaie, B. 2007. Domestication and crop physiology: Roots of green-revolution wheat. Ann. Bot. 100:991-998.

Wasson, A.P., Richards, R.A., Chatrath, R., Misra, S.C., Sai Prasad, S.V., Rebetzke, G.J., Kirkegaard, J.A., Christopher, J., Watt, M. 2012. Traits and selection strategies to improve root systems and water uptake in water-limited wheat crops. J. Exp. Bot. 63:3485-3498.

Watt, M., Schneebeli, K., Dong, P., Wilson, I.W. 2009. The shoot and root growth of Brachypodium and its potential as a model for wheat and other cereal crops. Funct. Plant Biol. 36:960-969.

White, P.J., George, T.S., Dupuy, L.X., Karley, A.J., Valentine, T.A., Wiese, L., Wishart, J. 2013. Root traits for infertile soils. Front. Plant Sci. 4:193.

Ytting, N.K., Andersen, S.B., Thorup-Kristensen, K. 2014. Using tube rhizotrons to measure variation in depth penetration rate among modern North-European winter wheat (Triticum aestivum L.) cultivars. Euphytica 199:233-245.

Zhu, J., Ingram, P.A., Benfey, P.N., Elich, T. 2011. From lab to field, new approaches to phenotyping root system architecture. Curr. Opin. Plant Biol. 14:310-317.

Zhu, J., Kaeppler, S.M., Lynch, J.P. 2005. Mapping of QTLs for lateral root branching and length in maize (Zea mays L.) under differential phosphorus supply. Theor. Appl. Genet. 111:688-695.

\section{Electronic Supplementary Material (ESM)}

Electronic Supplementary Material (ESM) associated with this article can be found at the website of CRC at http://www.akademiai.com/content/120427/

Electronic Supplementary Table S1. Provenance data and number of chromosomes of the wild populations and genotype Bd-21 of Brachypodium ssp. studied

Electronic Supplementary Table S2. Results of LSD tests for each of the eight variables with respect to: a) the type of PGS used; b) species of Brachypodium; c) the type of PGS used and species of Brachypodium; d) the genotypes of each Brachypodium species

Electronic Supplementary Figure S1. The plant growth support systems used: a) and d) gel chamber; b) and e) 'Termita' chamber; c) and f) Whatman paper. a), b) and c), before sowing the seeds. d), e) and f), after the period of development.

Electronic Supplementary Figure S2. Dendrogram based on the Euclidean distance of the means of the eight RSA characteristics analysed in the different genotypes of Brachypodium studied. Bd: Brachypodium distachyon; Bs: B. stacei and Bh: B. hybridum 